\title{
Estudo de tradução, adaptação e validação do ASSIST numa amostra de estudantes universitários portugueses
}

\author{
Sandra T. Valadas, Fernando R. Gonçalves \& Luís Faísca \\ Universidade do Algarve, Portugal
}

\begin{abstract}
Resumo
Neste estudo são analisadas as abordagens ao estudo, concepções de aprendizagem e preferências por diferentes estilos de ensino características de estudantes universitários. Foi nossa intenção construir uma versão portuguesa do Approaches and Study Skills Inventory for Students - short version (Tait, Entwistle \& McCune, 1998), aferindo a sua validade e fidelidade numa amostra de estudantes universitários portugueses. Os resultados na validade de constructo e os indicadores de fidelidade do instrumento parecem demonstrar que, no global, o ASSIST avalia os constructos teóricos subjacentes e os estudantes que integraram este estudo parecem, de facto, revelar determinadas formas de abordar as tarefas de estudo e de aprendizagem. São, contudo, de destacar algumas limitações, não só características de instrumentos desta natureza, mas também resultantes dos próprios procedimentos e de alguns resultados menos satisfatórios em algumas das subescalas do inventário.
\end{abstract}

Palavras-chave

Abordagens ao estudo; Concepções de aprendizagem; Estudantes universitários; ASSIST

\section{Introdução}

Neste artigo são analisados os constructos relativos às abordagens ao estudo, concepções de aprendizagem e preferências por diferentes estilos de ensino características dos estudantes universitários. 
Pela revisão da literatura percebemos que é considerável a diversidade de estudos internacionais que conduziram à construção de instrumentos de avaliação das concepções de aprendizagem e das abordagens ao estudo. A nível nacional são de referir algumas investigações sobre a temática específica das estratégias de estudo e de aprendizagem na Universidade: Bessa (2000, 2006), Bessa e Tavares (2000), Rosário (1999) e Rosário et al. (2000). Contudo, são ainda escassos (quando não inexistentes) os instrumentos, normalizados e validados, construídos especificamente para a população estudantil universitária portuguesa.

No estudo que se apresenta optámos por nos apoiar no trabalho de Entwistle e colaboradores que, neste domínio, assume particular relevância e protagonismo. A revisão teórica permitiu-nos encontrar um instrumento com características que se adequam aos nossos objectivos e utilizado em investigações internacionais deste âmbito. Referimo-nos ao Approaches and Study Skills Inventory for Students - short version (adiante designado ASSIST) (Tait et al., 1998) que, pelas provas de validade e fidelidade, nos pareceu ser o mais apropriado.

Foram objectivos principais construir uma versão portuguesa do ASSIST e aferir a sua validade e fidelidade numa amostra com características o mais semelhantes possível (ainda que, eventualmente, culturalmente divergentes) às dos estudos originais (realizados em Inglaterra). Sobre a relevância que assume a avaliação das características psicométricas, Field (2005) defende que um questionário deve obedecer a critérios de validade, fidelidade e discriminação. Tratando-se de uma adaptação para uma população (a portuguesa) com características e particularidades eventualmente distintas da população para a qual o instrumento foi originalmente construído (estudantes universitários ingleses), deverão pois ser considerados os critérios de validade e fidelidade do instrumento na amostra utilizada neste estudo.

Na medida em que a avaliação da validade é necessária, mas não suficiente para que um instrumento seja considerado de qualidade, apreciámos também a precisão das medidas por ele fornecidas, nomeadamente a consistência interna ou homogeneidade dos itens (indicador de fidelidade). 


\section{Instrumento: O Approaches and Study Skills Inventory for Students - short version (ASSIST)}

Para uma melhor compreensão do instrumento utilizado, não podemos deixar de contextualizar o seu aparecimento. O ASSIST constitui o último, de uma linha de inventários (o ASI - Approaches to Study Inventory - e o RASI - Revised Approaches to Study Inventory) desenhados para medir as diferenças individuais nas abordagens à aprendizagem dos estudantes do ensino superior (Diseth, 2001). O ASI foi desenvolvido por Entwistle e seus colegas na Universidade de Lancaster nos finais dos anos 70 e trata-se, de acordo com Richardson (1994c, 2000), do instrumento mais utilizado para avaliar a aprendizagem de estudantes no ensino superior. Em termos de estrutura, o instrumento foi construído com base nos resultados de estudos anteriores em que era explorada a aprendizagem dos estudantes: Hudson (1968), Marton e Säljö (1976a, 1976b), Biggs (1976, 1979) e Pask (1976). Ao longo dos anos, foram sendo efectuadas uma série de revisões do ASI original. Contudo, para alguns autores (Richardson, 2000; Tait et al., 1998), os instrumentos, de certa forma "emendados", comprometiam a integridade conceptual do ASI original, não possuindo algumas propriedades psicométricas desejáveis. O RASI (Entwistle, Tait \& McCune, 2000) continha inicialmente 32 itens que perfaziam um total de oito subescalas e três escalas: Abordagem Profunda, Abordagem Estratégica e Abordagem Superficial. Assim, no final dos anos 90, em resultado de um extenso trabalho de investigação, o instrumento foi revisto e designado de ASSIST. A primeira versão do ASSIST (Tait \& Entwistle, 1996) integrava 38 itens, construídos para identificar estudantes com estratégias de estudo pouco desejáveis. Nesta versão, o instrumento era constituído por subescalas que mediam quatro abordagens ao estudo, bem como a aptidão académica (definida como a auto-confiança académica). As quatro abordagens ao estudo seriam: Profunda (intenção de compreender, relacionar ideias, uso de dados e aprendizagem activa), Superficial (intenção de reproduzir, falta de compreensão, aprendizagem passiva e medo do fracasso), Estratégica (estudo organizado, gestão do tempo, atenção às exigências de avaliação e intenção de atingir a excelência) e Apática (ausência de objectivos e de interesse). Os estudantes respondiam a itens relacionados com estas abordagens numa escala tipo likert de cinco pontos, que ia desde "concordo" 
a "discordo". O resultado para cada uma das abordagens era obtido pela soma das pontuações dos respectivos itens.

Em termos globais, na versão mais recente do ASSIST, a Abordagem Profunda foi alargada de forma a incluir uma subescala de colaboração, que captava o desejo dos estudantes de discutirem com os seus pares; a definição de Abordagem Estratégica passou a integrar a subescala monitorização da eficácia, relacionada com a metacognição e a auto-regulação da aprendizagem; a Abordagem Superficial foi renomeada de Superficial Apática, colocando maior ênfase no estudo ineficaz; por fim, foi introduzida uma série de outras subescalas que os autores designaram de "motivos relacionados" (estratégias de aprendizagem, motivações e intenções que contribuem para as escalas). O ASSIST resulta assim de um desenvolvimento a partir do ASI, incluindo subescalas adicionais com vista a integrar a descrição dos processos relativos não só ao estudo, mas também às reacções ao ensino. $A$ versão mais recente mede as abordagens à aprendizagem em três dimensões ou escalas distintas: Profunda, Estratégica e Instrumental - esta última definida por Tait et al. (1998) como Superficial Apática.

São três as secções do instrumento: a primeira - o que é aprender? — remete para as concepções de aprendizagem descritas por Marton e Säljö (1976a, 1976b) e posteriormente desenvolvidas por Hattie, Biggs e Purdie (1996). Esta secção integra um conjunto de seis itens que avaliam a concepção do estudante sobre o que significa o termo aprender. Os itens funcionam como categorias que fazem parte, até certo ponto, de uma hierarquia. As primeiras três, numa extensão decrescente, tendem a relacionar-se com uma abordagem instrumental e podem ser combinadas para indicar uma concepção de aprendizagem enquanto reprodução de conhecimento (de natureza superficial) - subCA - Reprodutiva. As restantes três remetem para uma perspectiva de aprendizagem que envolve a compreensão e o desenvolvimento pessoais (de ordem mais profunda) subCA - Significativa. Os itens ou categorias que integram cada concepção são os que se seguem:

c. Aumentar o meu conhecimento através da obtenção de factos e informação.

a. Assegurar que me lembro bem das coisas que aprendo. 
d. Ser capaz de utilizar a informação adquirida.

e. Compreender material novo por mim mesmo(a).

f. Adquirir uma visão nova e mais significativa das coisas.

b. Contribuir para o meu desenvolvimento pessoal.

A segunda secção do inventário diz respeito às abordagens ao estudo, que resultam da perspectiva de Marton e Säljö (1976a, 1976b, 1997) sobre as abordagens à aprendizagem, em articulação com as descrições de Entwistle e Ramsden (1983) e Ramsden e Entwistle (1981) relativas a uma abordagem estratégica. Inclui 52 itens, reportados a três escalas (Profunda - escala AP, Estratégica - escala AE e Superficial Apática — escala ASA), que resultam da combinação de 13 subescalas. Cada subescala inclui quatro itens e cada escala (ou abordagem à aprendizagem) quatro ou cinco subescalas: a Abordagem Profunda inclui quatro subescalas, num total de 16 itens; na Abordagem Estratégica, são cinco as subescalas, totalizando 20 itens; e a escala Abordagem Superficial Apática reúne 16 itens em quatro subescalas distintas.

As três abordagens dividem-se em subescalas e subescalas de "motivos relacionados" - que remetem para estratégias de aprendizagem, motivações e intenções. Em cada abordagem, as primeiras três subescalas relacionam-se de uma forma mais consistente umas com as outras e podem ser combinadas com um certo grau de confiança para produzir medidas compósitas. As restantes subescalas e "motivos relacionados" (ou subescalas de motivação) têm maior probabilidade de variar nas suas inter-relações em função da diversidade de amostras utilizadas. Assim, de acordo com Entwistle (s/d) as relações entre subescalas necessitam de ser confirmadas sempre que se trate de uma amostra particular. Um aspecto que nos parece relevante referir diz respeito ao facto de os autores do instrumento considerarem as subescalas interesse por ideias (subll), realização (subR), monitorização da eficácia (subME) e medo do fracasso (subMF) como subescalas relacionadas (ou "motivos relacionados"), distinguindo-as, deste modo, das restantes. Este aspecto assume especial relevância em temos de análise. Em alguns estudos a que tivemos acesso, algumas das subescalas parecem agrupar-se, no sentido de darem um contributo mais significativo para a explicação das diferenças. Noutras investigações a que nos iremos referir, estas subescalas não foram consideradas nas análises. 
A terceira secção do instrumento remete para as preferências por diferentes tipos de aulas e de ensino. Trata-se de um conjunto de oito itens em que se pede ao estudante que indique até que ponto valoriza ou não diferentes tipos de aulas, exames, cursos e livros. Teoricamente, as respostas a estes itens reflectem dois factores latentes: suporte da compreensão e transmissão de informação, que correspondem às abordagens profunda e superficial apática respectivamente.

Por fim, uma última questão refere-se ao trabalho escolar já avaliado, numa escala de 1 (bastante mau) a 9 (muito bom). O estudante é aqui questionado sobre o seu aproveitamento e desempenho com base não só na sua auto-percepção, mas também no feedback recebido ao longo do semestre. Refira-se que esta última questão, designada de aproveitamento escolar, será sempre reportada à variável sucesso académico.

No que diz respeito aos procedimentos de cotação, as pontuações para as 13 subescalas resultam da soma das respostas individuais aos itens (de 1 a 5). As pontuações das três abordagens à aprendizagem (escalas) são o resultado dos valores obtidos nas subescalas que contribuem para cada abordagem ${ }^{1}$. Não houve itens introduzidos pelos autores do instrumento para controlo do enviesamento de resposta (sujeitos, portanto, a formulação negativa), pelo que não foi necessário reverter em termos de respostas.

Por último, refira-se que a aplicação do ASSIST pode ser individual ou colectiva e o tempo de aplicação pode ir de 25 a 45 minutos.

\section{Método}

\section{Amostra}

A amostra utilizada no nosso estudo integrava estudantes de uma universidade no sul de Portugal, tendo sido estratificada, no respeito por critérios de natureza teórica, em função do domínio ou área científica do curso de licenciatura e do ano frequentado.

Dos 626 estudantes inicialmente inquiridos, foram eliminados 60 questionários em virtude de não responderem à generalidade das questões (cinco sujeitos) e de serem alunos do $2^{\circ}$ (26 sujeitos) e do $3^{\circ}$ anos (29 sujeitos), apesar de frequentarem disciplinas dos anos em estudo. A amostra foi caracterizada em termos das variáveis ano, domínio científico e género. 
Tabela 1 - Distribuição da variável ano por domínio científico e género

\begin{tabular}{|c|c|c|c|c|c|c|c|c|c|c|c|c|}
\hline \multirow[b]{2}{*}{ Ano } & \multicolumn{2}{|c|}{ CHS } & \multicolumn{2}{|c|}{ CMA } & \multicolumn{2}{|c|}{ CT } & \multicolumn{2}{|c|}{ EG } & \multicolumn{2}{|c|}{ ERN } & \multicolumn{2}{|c|}{ Total } \\
\hline & M & $\mathbf{F}$ & M & $\mathbf{F}$ & M & $\mathbf{F}$ & $\mathbf{M}$ & $\mathbf{F}$ & M & $\mathbf{F}$ & $\mathbf{M}$ & $\mathbf{F}$ \\
\hline $1 . \circ$ ano & 15 & 56 & 12 & 21 & 24 & 15 & 41 & 44 & 9 & 18 & 101 & 154 \\
\hline 4.. ano & 4 & 68 & 22 & 36 & 52 & 40 & 10 & 20 & 18 & 22 & 106 & 186 \\
\hline 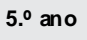 & - & 1 & 4 & 7 & 5 & - & - & - & 2 & - & 11 & 8 \\
\hline Total & 19 & 125 & 38 & 64 & 81 & 55 & 51 & 64 & 29 & 40 & 218 & 348 \\
\hline
\end{tabular}

Legenda: Ciências Humanas e Sociais (CHS), Ciências do Mar e do Ambiente (CMA), Ciências e Tecnologias (CT), Economia e Gestão (EG), Engenharia de Recursos Naturais (ERN).

Pela análise da tabela verificamos que a maioria $(\mathrm{N}=348)$ dos inquiridos é do género feminino (61.5\%). O domínio científico mais representado é o das Ciências Humanas e Sociais (25.4\%), enquanto o da Engenharia de Recursos Naturais é aquele que integra menor percentagem de estudantes (12.1\%). No que se refere à variável idade, os alunos inquiridos têm entre 18 e 48 anos $(M=22.29$; $D P=4.29$. $M o=21)$. Na altura da aplicação dos questionários, os 255 estudantes que frequentavam o $1^{\circ}$ ano apresentavam uma média etária de cerca de 20 anos $(M=20.57$; $D P=4.47)$, os do 4ํano ( $\mathrm{N}=292)$ uma média próxima dos 23 anos de idade $(\mathrm{M}=23.63$; $\mathrm{DP}=3.57)$ e os do $5^{\circ}$ ano $(\mathrm{N}=19)$ uma média de aproximadamente 25 anos $(\mathrm{M}=24.58$; $\mathrm{DP}=3.50)$. Os alunos que frequentavam os cursos da Faculdade de Ciências e Tecnologias eram, em média, mais velhos do que os estudantes das restantes faculdades $(\mathrm{M}=23.39)$.

\section{Procedimentos de recolha e análise de dados}

O processo de adaptação do ASSIST iniciou-se com o pedido ao autor do instrumento (Noel Entwistle - Universidade de Edimburgo), que prontamente autorizou a tradução e adaptação do mesmo. Respeitados os pedidos do autor - nomeadamente o compromisso de cedência da base de dados resultante da nossa investigação para estudos internacionais comparativos posteriores, bem como a não exclusão de itens do inventário -, procedemos à tradução do original inglês, que obedeceu ao método de 
tradução-retroversão (translate-translate back) (Hill \& Hill, 2000). O objectivo era obter uma tradução que correspondesse ao texto original, mas respeitando as particularidades características da língua portuguesa. A tradução do questionário para português foi realizada por sete indivíduos portugueses, todos com conhecimentos do inglês. Na comparação das versões recorremos aos esclarecimentos, anotações e orientações dos tradutores. A retroversão foi efectuada por um oitavo sujeito, português, com conhecimento das duas línguas (portuguesa e inglesa), residente num país anglo-saxónico durante alguns anos e com experiência de docência no ensino superior. Realizada a retroversão não se observaram quaisquer incompatibilidades com a tradução. Por último, procedemos ao refinamento da tradução, com vista a confirmar a adaptação lexical e semântica de cada item à língua portuguesa. De acordo com Cronbach (1984), estes procedimentos são legítimos, uma vez que contribuem para a melhoria da validade e da precisão do instrumento. Também Almeida e Freire (2000) referem que a consulta de especialistas constitui uma forma de análise qualitativa dos itens de um questionário e serve para apreciar o conteúdo e a forma dos itens em termos de clareza, compreensão e adequação aos objectivos do inventário (validade facial). Seguiu-se a adaptação das instruções (respeitando o mais possível as do inventário original), as quais orientavam o sujeito sobre a forma de preencher o instrumento. A versão portuguesa do ASSIST manteve a mesma estrutura da versão original: uma primeira parte relativa às concepções de aprendizagem, seguida das abordagens ao estudo e das preferências por diferentes tipos de aulas e de ensino, e por fim, uma questão relativa ao aproveitamento escolar.

Para recolha de dados, procedemos ao pedido de autorização aos diferentes responsáveis das Faculdades, cursos e disciplinas, tendo sido explicitados os objectivos da investigação, bem como garantida a confidencialidade da informação obtida. Foi solicitada a colaboração dos docentes, uma vez que os dados foram recolhidos em situação de aula normal.

As sessões de aplicação do instrumento realizaram-se sempre com a presença da investigadora, de forma a poder, quando necessário, atender aos pedidos de esclarecimento dos sujeitos. Os alunos eram sempre informados, verbalmente e através de um texto introdutório, dos objectivos do estudo, da confidencialidade das respostas e do carácter voluntário da sua participação. 
Além das análises qualitativas referidas anteriormente no momento da tradução, foram posteriormente utilizados procedimentos quantitativos que visam sobretudo apreciar parâmetros relativos à validade e à fidelidade do ASSIST. Numa abordagem preliminar das estatísticas descritivas do ASSIST, além do cálculo, para cada escala e subescala, da respectiva média e desviopadrão, procedemos à análise dos coeficientes de assimetria e de curtose. Seguiu-se a análise de validade de constructo do ASSIST, para a qual recorremos ao procedimento de análise factorial, com o objectivo de verificar se a estrutura factorial encontrada é comparável às outras estruturas resultantes de investigações anteriores com o inventário. Subjacentes aos procedimentos adoptados para a análise factorial, encontram-se tanto 0 objectivo de validação do instrumento como o de redução da variabilidade observada nas respostas a um número limitado de variáveis latentes (Field, 2005). Tendo em conta o número de sujeitos inquiridos considerados válidos para esta análise $(\mathrm{N}=566)$, a amostra do estudo de validação excede, em larga medida, o mínimo sugerido por vários autores de referência (Bryant \& Yarnold, 1995; Guilford, 1956; Gorsuch, 1983; Nunnally, 1978; Tabachnick \& Fidell, 1996). Optámos por realizar uma análise factorial exploratória para determinar a estrutura dos dados, utilizando, à semelhança de outros autores (Entwistle, Hanley \& Hounsell, 1979; Entwistle \& Ramsden, 1983; Diseth, 2002; Long, 2003), como método de extracção dos factores a análise de componentes principais (Principal Component Axis - PCA) ${ }^{2}$. O método de componentes principais é conhecido pela sua ampla utilização na determinação da estrutura factorial, na medida em que os seus resultados tendem a ser válidos (Stevens, 1986, cit. in Field, 2000) e fáceis de interpretar (Loewenthal, 2001, cit. in Maroco, 2003). Após o estudo da validade factorial, seguiu-se a análise dos índices de consistência interna das subescalas e escalas do ASSIST. Para a sua determinação, calculámos o coeficiente alfa de Cronbach para cada uma das subescalas e escalas resultantes da análise factorial. Tendo em vista a medida da homogeneidade dos itens, foram também calculados os coeficientes de correlação item-escala.

O nível de significância adoptado nos testes estatísticos efectuados foi de 0.05 . Todos os cálculos foram realizados com recurso ao programa de tratamento de dados estatísticos SPSS (versão 14.0). 


\section{Resultados e discussão}

Numa abordagem preliminar das estatísticas descritivas do ASSIST, além do cálculo, para cada escala e subescala, da respectiva média e desviopadrão, são também apresentados os coeficientes de assimetria e de curtose, bem como a amplitude (Tabela 2).

Tabela 2 - Estatísticas descritivas das Escalas e Subescalas do ASSIST

$$
(\mathrm{N}=566)
$$

\begin{tabular}{|lccccc|}
\hline Escalas/Subescalas & M & DP & Amplitude & Assimetria & Curtose \\
\hline Abordagem Profunda & 15.48 & 1.58 & $9-19$ & -0.30 & 0.66 \\
Procura de significado & 15.81 & 1.97 & $8-20$ & -0.38 & 0.43 \\
Relacionar ide ias & 14.89 & 2.12 & $7-20$ & -0.21 & 0.19 \\
Uso de dados & 15.99 & 1.83 & $9-20$ & -0.23 & 0.60 \\
Interesse por ideias & 15.26 & 2.18 & $8-20$ & -0.47 & 0.30 \\
Abordagem Superficial Apática & 12.22 & 2.11 & $7-19$ & 0.21 & -0.18 \\
Falta de objectivo & 10.12 & 3.48 & $4-20$ & 0.34 & -0.37 \\
Falta de compreensão & 10.98 & 2.74 & $4-20$ & 0.16 & -0.23 \\
Restrição ao programa & 12.66 & 2.84 & $5-20$ & 0.12 & -0.32 \\
Medo do fracasso & 15.11 & 2.72 & $6-20$ & -0.58 & 0.31 \\
Abordagem Estratégica & 14.30 & 1.86 & $9-19$ & -0.17 & 0.03 \\
Estudo organizado & 13.30 & 2.63 & $5-19$ & -0.27 & -0.21 \\
Gestão do tempo & 13.51 & 2.92 & $4-20$ & -0.29 & -0.02 \\
Atenção às exigências de avaliação & 14.16 & 2.31 & $6-20$ & -0.18 & -0.09 \\
Realização & 14.37 & 2.54 & $4-20$ & -0.46 & 0.87 \\
Monitorização da eficácia & 16.13 & 2.13 & $8-20$ & -0.60 & 0.83 \\
\hline
\end{tabular}

Uma primeira análise da tabela parece demonstrar que os estudantes pontuam, em termos médios, mais frequentemente na Abordagem Profunda, ainda que não possamos concluir sobre a significância desta diferença. Destacamos a pontuação média elevada obtida ao nível da subescala monitorização da eficácia - subME (M = 16.13).

No que respeita à amplitude, os sujeitos inquiridos utilizam a escala toda nas suas respostas (1 a 5), existindo pequenas variações das subescalas e escalas (entre quatro e 20). 
Quanto aos quocientes de assimetria ${ }^{3}$ e de curtose ${ }^{4}$, assumem, na generalidade dos casos, valores absolutos inferiores a zero (entre $-0.5 \mathrm{e}$ $0.5)^{5}$. Analisada a sua significância estatística (Skweness/Std Error; Kurtosis/ Std Error), os quocientes de assimetria assumiram, no geral, valores negativos e inferiores a -1.96 , não sendo significativos. Com base nos critérios referidos por Maroco (2003) e Pestana e Gageiro (2003) (valor inferior a 1.96 ou aproximadamente 2), rejeitamos portanto a simetria: estamos na presença, de uma distribuição assimétrica negativa que revela uma maior concentração de respostas nos níveis mais altos das escalas e menos dispersão dos níveis mais baixos. No que diz respeito aos valores da curtose, a distribuição não se apresenta normal.

\section{Validade de constructo}

À semelhança das análises factoriais realizadas noutras investigações (Byrne, Flood \& Willis, 2004b6; Diseth, 2001; Entwistle, Tait \& McCune, 2000; Kreber, 2003; Tait et al., 1998), neste estudo a análise factorial exploratória será feita sobre as subescalas e não sobre a totalidade dos itens. Refira-se que a análise factorial das subescalas é recomendada por Tait et al. (1998), bem como a associação entre os motivos relacionados e as escalas correspondentes. Sobre esta questão, Kreber (2003) chama a atenção para o facto de, quando os 52 itens foram sujeitos à análise de componentes principais, a solução de 13 factores sugerida por Tait e colaboradores (1998) não foi confirmada, o que conduziu a análises posteriores ao nível das subescalas e das escalas. Neste sentido, na presente análise serão criadas variáveis de segunda ordem resultantes da factorização das subescalas.

Recordamos que a versão do ASSIST utilizada neste estudo integra 52 itens que perfazem 13 subescalas e três escalas (Abordagem Profunda, Abordagem Estratégica e Abordagem Superficial Apática). Além destas, 0 inventário inclui itens que medem as orientações e concepções de aprendizagem (reprodutiva e significativa/transformativa) e as preferências por diferentes tipos de aulas e de ensino (suporte da compreensão e transmissão de informação). Os estudos anteriores não confirmaram a inclusão destas subescalas nas dimensões factoriais teoricamente descritas, motivo pelo qual optámos por seguir o mesmo procedimento. 
Apesar de não termos realizado uma análise factorial sobre os 52 itens, optámos por conduzir, além de análises item-subescala (para ilustrar a validade das subescalas) uma análise item-escala. Foi nossa intenção averiguar se os itens definidos a priori para cada escala correlacionavam entre si e com a respectiva escala, o que apoia a sua homogeneidade em termos dos constructos teóricos que pretendemos medir. Os coeficientes foram calculados com base nos itens individuais que contribuem para as diferentes subescalas e escalas. Ainda que a generalidade dos itens pareça correlacionar significativamente com a respectiva subescala, destacamos a existência de itens com coeficientes inferiores a 0.30 , o que pode indicar alguns problemas de homogeneidade.

A tabela que se segue permite uma visualização das correlações itemtotal por escala.

Tabela 3 - Coeficientes alfa de Cronbach, correlações item-total e medianas por escala

\begin{tabular}{|lccc|}
\hline Escalas & $\ldots$ & Item-total & Mediana \\
\hline Abordagem Profunda & 0.81 & $0.21-0.54$ & 0.45 \\
Abordagem Superficial Apática & 0.79 & $0.18-0.55$ & 0.43 \\
Abordagem Estratégica & 0.83 & $0.13-0.58$ & 0.44 \\
\hline
\end{tabular}

No geral, o inventário parece possuir uma homogeneidade adequada, com valores de correlação média entre 0.13 e 0.58 . No nosso estudo, a observação da matriz de correlações permitiu-nos constatar a existência de um número aceitável de coeficientes de correlação superiores a 0.30; na verdade, o teste de Bartlett foi significativo $(p<0.000)$, demonstrando que a matriz de correlações é distinta de uma matriz de identidade (Field, 2000). Este resultado, em conjunto com o índice de Kaiser-Meyer-Olkin (cujo valor pode ser classificado de muito bom, de acordo com Hutcheson e Sofroniou, 1999: KMO = 0.834), confirma a factoriabilidade dos dados, sendo a análise factorial considerada apropriada. Para determinar o número de componentes que seriam significativas na análise factorial, utilizámos o critério clássico de Kaiser (1960, cit. in Zwick \& Velicer, 1986), segundo o qual devem ser retidos 
os factores com valores próprios (eigenvalues) superiores a 1, bem como 0 scree plot de Cattell, que podemos observar na figura que se segue.

Figura 1 - Scree plot

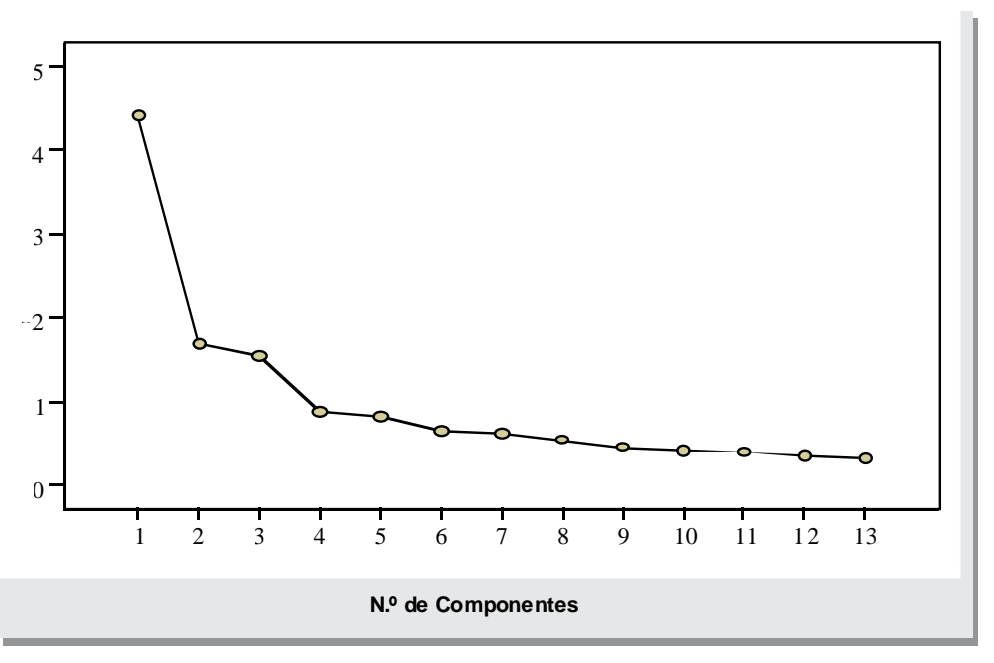

A análise do scree plot revela uma inflexão nítida após o terceiro componente, o que suporta a estrutura factorial de três componentes, tendo os restantes componentes valores próprios inferiores a 1. Tanto o critério de Kaiser como o scree plot indicam que deve ser extraída uma solução de três factores. Foi essa a nossa opção, procedendo em seguida à rotação dos três componentes retidos. Para ajudar a interpretar os factores encontrados utilizámos a rotação oblíqua (direct oblimin), por permitir que os factores se correlacionem (ou não) uns com os outros ${ }^{7}$. Na opinião de Duff (1997) uma rotação de natureza oblíqua é a opção certa para este inventário. Esta assunção encontra justificação nos estudos de Diseth (2001) e de Entwistle e colaboradores (2000), em que se observa uma dependência teórica entre os factores do ASSIST - este aspecto deve ser considerado quando escolhemos e método estatístico para validação do instrumento. A solução factorial encontrada explica aproximadamente $59 \%$ da variância total, com um contributo de $33.9 \%$ do componente 1 , de $13.0 \%$ do componente 2 e de $11.7 \%$ do componente 3. Para além dos critérios seguidos, esta solução parece ser 
a mais apropriada em termos de equilíbrio entre a interpretabilidade e a percentagem de variância explicada. Sobre valores desta ordem, Tinsley e Tinsley (1987) referem que é frequente a percentagem de variância explicada situar-se abaixo dos 50\%. Também Stevens (1986) afirma que, em certos contextos, se pode aceitar que cada factor explique, pelo menos, $5 \%$ da variância total, o que acontece em todos os factores encontrados. Atendendo a estas afirmações, a solução factorial encontrada parece ser satisfatória em termos da sua capacidade para explicar a variância total das respostas. A Tabela 4 permite uma melhor visualização dos resultados encontrados.

Tabela 4 - Correlações entre factores e sub-escalas e comunalidades $(\mathrm{N}=566)$

\begin{tabular}{|c|c|c|c|c|}
\hline \multirow[t]{2}{*}{ Subescalas } & \multicolumn{3}{|c|}{ Correlações } & \multirow[t]{2}{*}{ Comunalidades $^{*}$} \\
\hline & FI & F II & F III & \\
\hline \multicolumn{5}{|l|}{ Abordagem Profunda } \\
\hline Procura de significado & 0.79 & 0.04 & -0.03 & 0.61 \\
\hline Relacionar ideias & 0.79 & -0.03 & -0.05 & 0.60 \\
\hline Uso de dados & 0.78 & -0.01 & 0.12 & 0.70 \\
\hline Interesse por ideias & 0.68 & -0.10 & -0.01 & 0.49 \\
\hline \multicolumn{5}{|l|}{ Abordagem Superficial Apática } \\
\hline Falta de objectivo & -0.05 & 0.62 & 0.23 & 0.51 \\
\hline Falta de compreensão & -0.24 & 0.78 & 0.05 & 0.70 \\
\hline Restrição ao programa & -0.25 & 0.58 & -0.12 & 0.51 \\
\hline Medo do fracasso & 0.19 & 0.69 & 0.03 & 0.47 \\
\hline \multicolumn{5}{|l|}{ Abordagem Estratégica } \\
\hline Estudo organizado & -0.10 & -0.04 & 0.84 & 0.67 \\
\hline Gestão do tempo & -0.09 & -0.08 & 0.85 & 0.70 \\
\hline Atenção às exigências de avaliação & 0.18 & 0.31 & 0.53 & 0.41 \\
\hline Realização & 0.03 & -0.17 & 0.76 & 0.67 \\
\hline Monitorização da eficácia & 0.35 & -0.01 & 0.51 & 0.53 \\
\hline \multicolumn{5}{|l|}{ Correlações entre factores } \\
\hline Factor I & 1.00 & -0.13 & 0.37 & \\
\hline Factor II & -0.13 & 1.00 & -0.16 & \\
\hline Factor III & 0.37 & -0.16 & 1.00 & \\
\hline
\end{tabular}

* O coeficiente de comunalidade (h2) dá-nos informações sobre a proporção de variância de cada item que pode ser explicada pelos factores extraídos, isto é, em que medida cada item se relaciona com os factores. 
Os três factores obtidos após rotação são os esperados conceptualmente e podem ser claramente identificados como profundo (Factor I), superficial apático (Factor II) e estratégico (Factor III). Tal como previsto teoricamente, observámos uma correlação positiva entre o primeiro (Abordagem Profunda) e o terceiro (Abordagem Estratégica) factor, o que indica que os factores não são totalmente independentes entre si. Na verdade, ainda que a investigação sobre as abordagens tenha vindo a demonstrar que as pontuações nas escalas Abordagem Profunda e Abordagem Superficial são relativamente independentes (correlacionando negativamente umas com as outras), é frequente a Abordagem Profunda correlacionar significativamente com a Abordagem Estratégica. De uma forma não surpreendente, Entwistle, Tait e McCune (2000) relataram uma correlação positiva moderada entre os factores profundo e estratégico, uma correlação negativa entre a abordagem profunda e a abordagem superficial apática e a abordagem estratégica e a abordagem superficial apática. Resultados semelhantes foram observados no presente estudo. Neste sentido, na análise das pontuações obtidas pelos sujeitos, deve ser possível que os factores se correlacionem - o que justifica a opção pela rotação oblíqua (direct oblimin rotation ${ }^{8}$ ).

No que diz respeito às correlações das subescalas com os factores, Kline (1994), Loewenthal (2001) e Nunnally (1978) recomendam a inclusão de itens com saturação igual ou superior a 0.30 - critério também adoptado por Entwistle e colaboradores (2000). Foi este o critério utilizado no nosso estudo.

De referir uma particularidade, comum a outras investigações com o ASSIST: duas subescalas (monitorização da eficácia (subME) e atenção às exigências de avaliação (subAEAV)) saturam simultaneamente em dois componentes $^{9}$. Relativamente às particularidades observadas nas subescalas monitorização da eficácia (subME) e atenção às exigências de avaliação (subAEAV), o facto de uma mesma subescala pontuar em duas escalas é consistente com os resultados de Byrne e colaboradores (1999, 2004a) e Diseth (2001).

No que se refere aos valores das comunalidades, de acordo com Field (2005) estas devem ser sempre superiores a 0.5 após a extracção. Pela análise da tabela verificamos que, à excepção de três das 13 subescalas, todas assumem valores superiores ou muito próximos de 0.5 , pelo que os valores são considerados aceitáveis (Stevens, 1986). 
Quanto aos resultados obtidos na análise factorial parecem-nos pertinentes algumas considerações sobre outros estudos com o ASSIST, porque permitem uma melhor compreensão dos procedimentos utilizados. Encontrámos na literatura sobre os instrumentos de avaliação das abordagens construídos pelo Grupo de Lancaster vários estudos de validação quer de versões anteriores do ASI, quer do RASI e do ASSIST. A versão mais antiga (o ASI) apresentava frequentemente alguns problemas em reproduzir a estrutura factorial. Harper e Kember (1989) e Richardson (1994a, 1994b), por exemplo, chamaram a atenção para o facto de o ASI possuir validade e fidelidade limitadas. Entwistle e Waterson (1988), Meyer e Parsons (1989) e Speth e Brown (1988) também não conseguiram a reprodução da estrutura factorial do ASI, particularmente do que diz respeito à subescala realização e às escalas de estilos e patologias de aprendizagem, o que sugeria uma validade de constructo limitada. Noutros estudos sobre a consistência interna das 16 subescalas que integravam originalmente o instrumento, foram encontrados coeficientes alfa entre 0.29 e 0.78 (Entwistle \& Ramsden, 1983).

Quanto aos componentes, Harper e Kember (1989) conseguiram reproduzir a dicotomia profunda/superficial, mas outros dois factores - de realização e orientação não-académica - apresentavam alguma instabilidade, possivelmente devido à influência do contexto. Pelo contrário, os estudos de Richardson (1990) e de Sadler-Smith (1996) suportaram a estrutura factorial do ASI. Outras investigações centraram-se nos dois grandes factores relativos à orientação significativa (profunda) e reprodutiva (superficial), tendo na base uma versão abreviada do inventário (Duff, 1997). Utilizando esta versão do ASI, Newstead (1992), por exemplo, enfatizou as suas vantagens enquanto ferramenta de investigação e encontrou níveis moderados de fidelidade. Os factores conceptualmente previstos acabaram por surgir durante a análise de dados, o que, de acordo com Riding e Rayner (1998), confirmava a validade preditiva da medida.

No nosso estudo estamos na presença de uma estrutura com três componentes que revelam saturações tendencialmente fortes, consistente com a investigação original realizada pelos autores do inventário, bem como com os resultados de estudos posteriores (Byrne et al., 2002, 2004a, 2004b; Diseth, 2001, 2002; Entwistle, Tait \& McCune, 2000; Kreber, 2003; Long, 2003; Tait et al., 1998). Neste sentido, o modelo de três factores parece produzir um ajustamento apropriado. 


\section{Consistência interna do instrumento}

De seguida, apresentamos a análise da consistência interna das escalas e subescalas do ASSIST, por nós identificadas pela análise factorial. Chamamos a atenção para o facto de não ser possível falar na existência de um constructo psicológico único subjacente ao ASSIST. Na verdade, trata-se de um inventário multidimensional, pelo que não faz sentido juntar os resultados das diferentes subescalas num resultado compósito global.

No seguimento da recomendação de Duff (2001) de que cada estudo proporcione evidências da consistência interna dos dados, importa olhar para os resultados obtidos no nosso estudo, comparando-os com investigações realizadas noutros países. A Tabela 5 apresenta os valores comparativos.

\section{Tabela 5 - Coeficientes alfa de Cronbach $(\alpha)$ por escala e subescala}

\begin{tabular}{|c|c|c|c|c|c|c|}
\hline & $\begin{array}{c}\text { Portugal } \\
(\mathrm{N}= \\
566)\end{array}$ & $\begin{array}{l}\text { E.U.A. } \\
(N= \\
298)\end{array}$ & $\begin{array}{c}\text { Irlanda } \\
(\mathbf{N}= \\
437)\end{array}$ & $\begin{array}{c}\begin{array}{c}\text { Noruega } \\
(N= \\
573)\end{array}\end{array}$ & $\begin{array}{c}\text { Canadá } \\
(N= \\
1080)\end{array}$ & $\begin{array}{c}\text { Reino } \\
\text { Unido } \\
(\mathrm{N}= \\
817)\end{array}$ \\
\hline Abordagem Profunda & 0.81 & 0.82 & 0.84 & 0.81 & - & 0.84 \\
\hline Procura de significado & 0.51 & 0.55 & 0.63 & 0.49 & 0.62 & 0.57 \\
\hline Relacionar ideias & 0.54 & 0.59 & 0.59 & 0.62 & 0.59 & 0.59 \\
\hline Uso de dados & 0.59 & 0.49 & 0.59 & 0.49 & 0.51 & 0.53 \\
\hline Interesse por ideias & 0.56 & 0.67 & 0.69 & 0.64 & 0.73 & 0.76 \\
\hline Abordagem Superficial & 0.79 & 0.80 & 0.83 & 0.70 & - & 0.87 \\
\hline Apática & 0.54 & 0.57 & 0.59 & 0.68 & 0.60 & 0.57 \\
\hline Falta de compreensão & 0.73 & 0.68 & 0.75 & 0.57 & 0.72 & 0.76 \\
\hline Falta de objectivo & 0.62 & 0.55 & 0.64 & 0.57 & 0.59 & 0.55 \\
\hline \multirow{2}{*}{$\begin{array}{l}\text { Restrição ao programa } \\
\text { Medo do fracasso }\end{array}$} & 0.63 & 0.72 & 0.74 & 0.57 & 0.75 & 0.69 \\
\hline & 0.83 & 0.87 & 0.87 & 0.81 & - & 0.80 \\
\hline Abordagem Estratégica & 0.51 & 0.55 & 0.63 & 0.59 & 0.59 & 0.54 \\
\hline Estudo organizado & 0.65 & 0.77 & 0.74 & 0.72 & 0.80 & 0.68 \\
\hline \multicolumn{7}{|l|}{ Gestão do tempo } \\
\hline Atenção às exigências & 0.40 & 0.56 & 0.63 & 0.41 & 0.62 & 0.76 \\
\hline de avaliação & 0.67 & 0.63 & 0.68 & 0.66 & 0.67 & . \\
\hline $\begin{array}{l}\text { Rea lização } \\
\text { Monitorização da } \\
\text { eficácia }\end{array}$ & 0.58 & 0.61 & 0.61 & 0.51 & 0.60 & 0.62 \\
\hline
\end{tabular}

Almeida e Freire (2000) e Loewenthal (2001) consideram que coeficientes iguais ou superiores a 0.70 são aceitáveis. Autores como Kline (1994) afirmam que quando lidamos com constructos psicológicos, são de esperar valores inferiores a 0.70 devido à diversidade dos constructos que estão a ser medidos. Se o valor encontrado for ligeiramente superior, tal não 
significa necessariamente que a escala é homogénea: no presente estudo sabemos que não é, uma vez que o questionário contém um número significativo de subescalas, as quais não podemos esperar que sejam necessariamente consistentes. Assim, ainda que alguns valores respeitantes às subescalas sejam inferiores ao valor do total, tal justifica-se considerando os respectivos números de itens (cada escala integra apenas quatro). Também no nosso estudo se observam valores baixos ao nível da subescala atenção às exigências de avaliação $(\alpha=0.40)$, ainda que o valor obtido não difira grandemente nos outros estudos referidos. Os valores obtidos para a amostra estudada, quer para as escalas quer para as subescalas, são aceitáveis para escalas desta natureza (Entwistle et al., 2000), sendo próximos dos obtidos em outros estudos com o ASSIST (Byrne et al., 1999, 2004a; Tait et al., 1998; Entwistle et al., 2000; Diseth, 2001). Contudo, não podemos deixar de recomendar alguma precaução na interpretação dos resultados.

\section{Conclusões}

Tal como afirmámos inicialmente, foram objectivos principais construir uma versão portuguesa do ASSIST e aferir a sua validade e fidelidade, considerando que se trata de uma adaptação para uma população com características e particularidades distintas da população para a qual o instrumento foi originalmente construído.

Com base na revisão da literatura, vimos que o ASSIST resulta de um desenvolvimento a partir do ASI, incluindo subescalas adicionais com vista a integrar a descrição dos processos relativos não só ao estudo, mas também às reacções ao ensino. Neste momento, podemos afirmar com alguma segurança que as versões mais recentes (o RASI e o ASSIST) parecem possuir as propriedades psicométricas apropriadas para instrumentos desta natureza. Refira-se o estudo com a versão reduzida do RASI de Duff (1997), que classificou o instrumento como robusto na avaliação das grandes diferenças entre uma abordagem profunda, uma superficial e uma outra estratégica. Na mesma linha, Entwistlle e colaboradores (2000) obtiveram resultados com o ASSIST que suportam a assunção de uma solução de três factores (profundo, superficial e estratégico). Também Diaz (1984, cit. in Entwistle, 1988), utilizando a versão espanhola do ASI, encontrou os mesmos 
factores, o que indica alguma consistência transcultural. Sobre esta questão da transculturalidade, Richardson (1995) alerta para a existência de diferenças qualitativas nas motivações para estudar no ensino superior em função do contexto em causa. No seu entender as abordagens ao estudo são específicas da cultura, pelo que devemos ser cautelosos com o uso destes instrumentos em culturas não ocidentais.

Tendo em conta os resultados apresentados neste artigo, as medidas do ASSIST parecem reflectir os constructos que pretendem medir - o que constitui um indicador de validade de constructo. Os três factores obtidos são os esperados conceptualmente e podem ser claramente identificados como profundo (Factor I), superficial apático (Factor II) e estratégico (Factor III). Destacamos, uma vez mais que, tal como previsto teoricamente (Entwistle et al., 2000), os factores Abordagem Profunda e Abordagem Estratégica não são totalmente independentes entre si. Não podemos deixar de referir ainda a saturação em simultâneo de duas subescalas (monitorização da eficácia e atenção às exigências de avaliação) em dois componentes distintos. Tal como observámos, trata-se de uma particularidade comum a outras investigações com o ASSIST (Byrne et al., 1999, 2004a; Diseth, 2001). Entwistle e colaboradores (2000) defendem mesmo que é totalmente compreensível em termos conceptuais, considerando a subescala atenção às exigências de avaliação como mais relevante para estudantes de anos terminais. Adicionalmente, Entwistle e McCune (2004) acrescentam que a existência de uma certa relação entre domínios não deve ser entendida como um aspecto negativo, mas antes como uma inevitabilidade inerente ao comportamento humano. Um outro aspecto a destacar é o facto de se tratar de "motivos relacionados" e não propriamente escalas de medida dos constructos, sendo de esperar que estas subescalas relacionadas saturem pior do que as subescalas ditas "puras".

Em termos de precisão das medidas fornecidas pelo ASSIST, encontrámos indicadores de consistência interna satisfatórios nas três escalas do instrumento, ainda que alguns dos valores difiram dos observados noutros estudos. Assim, sugerimos alguma precaução na interpretação dos resultados. Não obstante, na análise das correlações item-total foram observados valores bastantes baixos. Em rigor metodológico, seria desejável a exclusão destes itens. Contudo, realçamos que uma eventual 
reformulação/eliminação deverá sempre obedecer a critérios teóricos que presidiram à construção do inventário, os quais ultrapassam, amplamente, os objectivos deste trabalho.

Por se tratar de um inventário com um número de itens bastante aceitável, a sua utilização assume-se vantajosa. Globalmente, realçamos a composição, características psicométricas e indicadores de validade satisfatórios do ASSIST, próximos dos encontrados em outros estudos. Ainda que reconhecendo a necessidade de prosseguirmos com novas análises, parece-nos possível a sua utilização isoladamente, como medida de diagnóstico das abordagens ao estudo e concepções de aprendizagem de estudantes do ensino superior. Defendemos, à semelhança de outros autores (Byrne et al., 2004a), que o instrumento falha, até certo ponto, na captação da complexidade das formas individuais de aprender e de estudar. Assim, tendo em vista explorar a riqueza individual da aprendizagem dos estudantes, a proposta é a de utilizar métodos mistos de investigação, que combinem abordagens quantitativas e qualitativas. Recomenda-se, portanto, o uso de amostras de estudantes pertencentes a mais do que uma instituição universitária, tendo em vista captar a variação inter e intraindividual. Nesta linha de pensamento, as limitações encontradas proporcionam também uma oportunidade para, em investigações futuras, se aplicar o instrumento em amostras adicionais, o que torna possível ainda a análise das subescalas que não se comportam exactamente como se esperava teoricamente (nomeadamente as subescalas atenção às exigências de avaliação e monitorização da eficácia).

\section{Notas}

1 Assume-se que cada item se comporta como uma variável (por exemplo P04 = item Profundo 4). O total numa subescala resulta assim da criação de uma nova variável pela soma dos itens. Por exemplo, a subescala procura de significado $($ subPS) $=$ $\mathrm{P} 04+\mathrm{P} 17+\mathrm{P} 30+\mathrm{P} 43$. A Abordagem Profunda (escala AP) seria = PS + RI + UD + II.

Uma vez que são quatro as subescalas que integram a escala $A P$ e cinco as subescalas que contribuem para a escala $\mathrm{AE}$, para uma compreensão mais fácil, optámos, à semelhança de outros investigadores, por dividir cada escala pelo número de subescalas constituintes, com vista a estandardizar as pontuações, que ficam assim circunscritas a um mínimo de quatro e a um máximo de 20 pontos. 
2 Diseth (2001), ao realizar uma análise factorial exploratória do instrumento, optou pelo método principal axis factoring (PAF), com rotação oblíqua dos factores. A PAF tenta explicar a variação partilhada pela variável em causa e que se espera ser, por este facto, mais fiel. Outros autores (Byrne, Flood \& Willis, 2004a; Entwistle, McCune \& Walker, 2001) optaram pela maximum likelihood extraction para analisar as subescalas.

3 Este quociente é utilizado para não rejeitar a simetria, o que acontece se o seu resultado for menor que 1.96 (ou aproximadamente 2) em valor absoluto (Pestana \& Gageiro, 2003).

4 Trata-se de uma medida de achatamento que fornece outra característica da forma da distribuição, através da comparação com uma distribuição normal, para um dado desvio padrão, indicando a intensidade das frequências à volta de um ponto central (Pestana \& Gageiro, 2003).

5 Quer o coeficiente de assimetria, quer o de achatamento (curtose) são geralmente utilizados para comparar a forma da distribuição em estudo com uma distribuição teórica de uso ubíquo em inferência estatística (a distribuição normal). De acordo com Maroco (2003), para que uma distribuição se possa assumir como normal, os valores dos coeficientes descritos devem ser próximos de zero (i.e. dentro de um intervalo de ] - 0.5;0.5 [ ). Sempre que os valores absolutos destes coeficientes sejam superiores a 1, pode assumir-se que a distribuição de dados em causa não é do tipo normal.

6 Byrne et al. (2004b) conduziram uma análise factorial ao nível dos itens. O resultado mostrou pouca variação em relação à solução com base nas subescalas, suportando os três factores obtidos.

7 Sobre o uso deste tipo de rotação, Everitt (1996) alerta para o facto de correlações substanciais entre factores poderem tornar a interpretação de uma solução oblíqua mais difícil do que uma solução ortogonal correspondente (p. 236).

8 Não obstante, importa referir que quer Byrne, Flood e Willis (2004b) quer Entwistle, McCune e Walker (2001) realizaram uma análise factorial das subescalas com extracção pelo método maximum likelihood (que força uma solução ortogonal que pressupõe a independência dos factores).

9 Smith, Miller e Crassini (1998) defendem que quando uma subescala pontua substancialmente em mais do que um factor e a diferença entre os valores das saturações é superior a 0.20 , é considerado distintivo o valor mais alto.

\section{Referências}

ALMEIDA, Leandro S. \& FREIRE, Teresa (2000). Metodologia da Investigação em Psicologia e Educação. Braga: Psiquilíbrios.

BESSA, José A. (2000). Níveis de ajustamento e auto-regulação académica em esudantes universitários. Dissertação de Mestrado. Aveiro: Universidade de Aveiro. 
212 Sandra T. Valadas, Fernando R. Gonçalves \& Luís Faísca

BESSA, José A. (2006). (Des)Continuidades na transição entre secundáro e superior: efeitos das experiências (pessoais e contextuais) do 'caloiro' universitário na auto-reglação e rendimento académicos. Tese de Doutoramento. Aveiro: Universidade de Aveiro.

BESSA, José A. \& TAVARES, José (2000). Abordagens e estratégias de regulação do estudo em alunos do 1ำ ano das licenciaturas de ciências e engenharia da Universidade de Aveiro. Psicologia, vol XIV(2), pp.173-188.

BIGGS, John (1976). Dimensions of study behaviour: another look at A.T.I. British Journal of Educational Psychology, vol 46(1), pp. 68-80.

BIGGS, John (1979). Individual differences in study processes and the quality of learning process. Higher Education, vol 8, pp. 381-394.

BRYANT, Fred B. \& YARNOLD, Paul R. (1995). Principal component analysis and exploratory and confirmatory factor analysis. In L. Grimm \& P. Yarnold (Eds.), Reading and Understanding Multivariate Analysis. Washington, DC: American Psychological Association Books, pp. 99-136.

BYRNE, Marann; FLOOD, Barbara \& WILLIS, Pauline (1999). Approaches to Learning: Irish students of accounting. Irish Accounting Review, vol 6(2), pp. 1-29.

BYRNE, Marann; FLOOD, Barbara \& WILLIS, Pauline (2002). The relationship between learning approaches and learning outcomes: a study of Irish accounting students. Accounting Education: an International Journal, vol 11(1), pp. 27-42.

BYRNE, Marann; FLOOD, Barbara \& WILLIS, Pauline (2004a). Validation of the Approaches and Study Skills Inventory for Students (ASSIST) using accounting Students in the USA and Ireland: a research note. Accounting Education: an International Journal, vol 13(4), pp. 449-459.

BYRNE, Marann; FLOOD, Barbara \& WILLIS, Pauline (2004b). Using the Student Learning Framework to Explore the Variation in Academic Performance of European Business Students. Journal of Further and Higher Education, vol 28(1), pp. 67-78.

CRONBACH, Lee J. (1984). Essentials of Psychological Testing (4th ed.), New York: Harper Row.

DISETH, Åge (2001). Validation of a Norwegian version of the Approaches and Study Skills Inventory for Students (ASSIST): an application of structural equation modelling. Scandinavian Journal of Educational Research, vol 45(4), pp. 381394.

DISETH, Åge (2002). The Relationship between Intelligence, Approaches to Learning and Academic Achievement. Scandianavian Journal of Educational Research, vol 46(2), pp. 219-230.

DUFF, Angus (1997). A note on the reliability and validity of a 30-item version of Entwistle \& Tait's Revised Approaches to Learning Inventory. British Journal of Educational Psychology, vol 67(4), pp. 529-539.

DUFF, Angus (2001). A note on the psychometric properties of the Learning Styles Questionnaire (LSQ). Accounting Education, vol 10(2), pp. 185-197.

ENTWISTLE, Noel (s/d). Scoring Key for the Approaches and Study Skills Inventory for Students (ASSIST). [Em linha] [Acedido em 22 de Fevereiro de 2006, disponível em http://www.tla.ed.ac.uk/etl/questionnaires/ASSIST.pdf.]. 
ENTWISTLE, Noel (1988). Styles of Learning and Teaching. London: David Fulton.

ENTWISTLE, Noel; HANLEY, Maureen \& HOUNSELL, Dai (1979). Identifying distinctive approaches to studying. Higher Education, vol 8, pp. 365-380.

ENTWISTLE, Noel \& MCCUNE, Velda (2004). The conceptual bases of study strategy inventories in higher education. Educational Psychology Review, vol 16(4), pp. 325-345.

ENTWISTLE, Noel \& RAMSDEN, Paul (1983). Understanding Student Learning. London: Croom Helm.

ENTWISTLE, Noel; MCCUNE, Velda \& WALKER, Paul (2001). Conceptions, styles and approaches within higher education; Analytical abstractions and everyday experience. In R. Sternberg \& Li-F. Zhang (Eds.), Perspectives on Thinking, Learning, and Cognitive Styles. Mahwah, NJ, US: Lawrence Erlbaum Associates Inc., Publishers, pp. 103-136.

ENTWISTLE, Noel; TAIT, Hilary \& MCCUNE, Velda (2000). Patterns of response to an approaches to studying inventory across contrasting groups and contexts. European Journal of Psychology of Education, vol 15(1), pp. 33-48.

ENTWISTLE, Noel J. \& WATERSON, S. (1988). Approaches to studying and levels of processing in university students. British Journal of Educational Psychology, vol 58(3), pp. 258-265.

EVERITT, Brian (1996). An Introduction to finite mixture distribution. Statistical Methods in Medical Research, vol 5, pp. 107-127.

FIELD, Andy (2000). Discovering Statistics Using SPSS. London: Sage Publications.

FIELD, Andy (2005). Discovering Statistics Using SPSS. London: Sage Publications.

GUILFORD, Joy P. (1956). Psychometric Methods. New York: McGraw-Hill.

GORSUCH, Richard L. (1983). Factor Analysis. Hillsdale, NJ: Lawrence Erlbaum.

HARPER, Greg \& KEMBER, David (1989). Interpretation of factor analyses from the Approaches to Studying Inventory. British Journal of Educational Psychology, vol 59, pp. 66-74.

HATTIE, John; BIGGS, John \& PURDIE, Nola (1996). Effects of learning skills interventions on student learning: A meta-analysis. Review of Educational Research, vol 66(2), pp. 99-136.

HILL, Manuela M. \& HILL, Andrew (2000). Investigação por Questionário. Lisboa: Edições Sílabo, Lda.

HUDSON, Liam (1968). Frames of Mind: Ability, Perception and Self-Perception in the Arts and Sciences. London: Methuen.

HUTCHESON, Graeme \& SOFRONIOU, Nick (1999). The Multivariate Social Scientist: Introductory Statistics Using Generalized Linear Models. Thousand Oaks, CA: Sage Publications.

KLINE, Paul (1994). An Easy Guide to Factor Analysis. London: Routledge.

KLINE, Paul (2000). Handbook of Psychological Testing. New York: Routledge.

KREBER, Carolin (2003). The scholarship of teaching: A comparison of conceptions held by experts and regular academic staff. Higher Education, vol 46, pp. 93-121. 
LOEWENTHAL, Kate (2001). An Introduction to Psychological Tests and Scales. Hove, East Sussex: Psychology Press Ltd.

LONG, William F. (2003). Dissonance detected by cluster analysis of responses to the approaches and study skills inventory for students. Studies in Higher Education, vol 28(1), pp. 21-35.

MAROCO, João (2003). Análise Estatística com Utilização do SPSS (2ª edição). Lisboa: Edições Sílabo, Lda.

MARTON, Ference \& SÄLJÖ, Roger (1976a). On qualitative differences in learning, I Outcome and Process. British Journal of Educational Psychology, vol 46(1), pp. 4-11.

MARTON, Ference \& SÄLJÖ, Roger (1976b). On qualitative differences in learning, II Outcome as a function of the learner's conception of the task. British Journal of Educational Psychology, vol 46(1), pp. 115-127.

MARTON, Ference \& SÄLJÖ, Roger (1997). Approaches to learning. In F. Marton; D.J. Hounsell \& N.J. Entwistle (Eds.), The Experience of Learning: Implications for teaching and Studying in Higher Education (2nd ed.). Edinburgh: Scottish Academic Press, pp. 39-58.

MEYER, Jan H. \& PARSONS, Phillip G. (1989). Approaches to studying and course perceptions using the Lancaster Inventory: a comparative study. Studies in Higher Education, vol 14(2), pp. 137-153.

NEWSTEAD, Stephen E. (1992). A study of two "quick-and-easy" methods of assessing individual differences in student learning. British Journal of Educational Psychology, vol 62, pp. 299-312.

NUNNALLY, Jum C. (1978). Psychometric Theory. (2nd ed.). New York: McGraw Hill.

PASK, Gordon (1976). Styles and strategies of learning. British Journal of Educational Psychology, vol 46, pp. 128-148.

PESTANA, Maria H. \& GAGEIRO, João N. (2003). Análise de Dados para Ciências Sociais: A Complementaridade do SPSS ( $3^{a}$ edição). Lisboa: Edições Sílabo, Lda.

RAMSDEN, Paul \& ENTWISTLE, Noel (1981). Effects of academic departments on students' approaches to studying. British Journal of Educational Psychology, vol 51, pp. 368-383.

RICHARDSON, John T. (1990). Reliability and replicability of the Approaches to Studying Questionnaire. Studies in Higher Education, vol 15(2), pp. 155-168.

RICHARDSON, John T. (1994a). Mature students in higher education: academic performance and intellectual ability. Higher Education, vol 28(3), pp. 373-386.

RICHARDSON, John T. (1994b). Mature students in higher education: 1. A literature survey on approaches to studying. Studies in Higher Education, vol 19(3), pp. 309-325.

RICHARDSON, John T. (1994c). Cultural specificity of approaches to studying higher education: A literature survey. Higher Education, vol 27, pp. 449-468.

RICHARDSON, John T. (1995). Cultural specificity of approaches to studying in higher education: a comparative investigation using the Approaches to Studying Inventory. Educational and Psychological Measurement, vol 55(2), pp. 300-309. 
RICHARDSON, John T. (2000). Researching Student Learning: Approaches to Studying in Campus-based and Distance Learning. Buckingham: SHRE and Open University Press.

RICHARDSON, John T. (2004). Methodological Issues in Questionnaire-Based Research on Student Learning in Higher Education. Educational Psychology Review, vol 16(4), pp. 347-358.

RIDING, Richard J. \& RAYNER, Steve (1998). Cognitive Styles and Learning Strategies. London: Fulton.

ROSÁRIO, Pedro L. (1999). As Abordagens dos Alunos ao Estudo: Diferentes Modelos e suas Interrelações. Teoria, Investigação e Prática, vol 4(1), pp. 43-61.

ROSÁRIO, Pedro L.; ALMEIDA, Leandro S.; GUIMARÃES, Carina; FARIA, Ana; PRATA, Lúcia; DIAS, Mónica \& NÚÑEZ, Carlos (2000). As abordagens dos alunos à aprendizagem em função da área académica: Uma investigação na Universidade do Minho. In A.P. Soares; A. Osório; J. V. Capela; L. S. Almeida; R. M. Vasconcelos \& S. Caires (Eds.), Transição para o Ensino Superior. Braga: Universidade do Minho, Conselho Académico, pp. 133-145.

SADLER-SMITH, Eugene (1996). Approaches to studying: age, gender and academic performance. Educational Studies, vol 22(3), pp. 367-379.

SMITH, Swee N.; MILLER, Robyn J. \& CRASSINI, Boris (1998). Approaches to studying of Australian and overseas Chinese university students. Higher Education Research and Development, vol 17(3), pp. 261-276.

SPETH, Carol \& BROWN, Robert (1988). Study approaches, processes and strategies: are three perspectives better than one? British Journal of Educational Psychology, vol 58(3), pp. 247-257.

STEVENS, James (1986). Applied Multivariate Statistics for the Social Sciences. (1st edition). New Jersey: Lawrence Erlbaum Associates.

TABACHNICK, Barbara G., \& FIDELL, Linda S. (1996). Using Multivariate Statistics. (3rd ed.). New York: Harper Collins.

TAIT, Hilary \& ENTWISTLE, Noel (1996). Identifying students at risk through ineffective study strategies. Higher Education, vol 31(1), pp. 97-116.

TAIT, Hilary; ENTWISTLE, Noel \& MCCUNE, Velda (1998). ASSIST: a reconceptualisation of the Appoaches to Studying Inventory. In C. Rust (ed.), Improving Student Learning: Improving Students as Learners. Oxford: Oxford Brookes University, Oxford Centre for Staff and Learning Development.

TINSLEY, Howard E. A. \& TINSLEY, Diane J. (1987). Uses of factor analysis in counseling psychology research. Journal of Counseling Psychology, vol 34, pp. 414-424.

ZWICK, William R. \& VELICER, Wayne F. (1986). Comparison of five rules for determining the number of components to retain. Psychological Bulletin, vol 3 , pp. 432-442. 
216 Sandra T. Valadas, Fernando R. Gonçalves \& Luís Faísca

TRANSLATION, ADAPTATION AND VALIDATION STUDY OF THE ASSIST IN A SAMPLE OF PORTUGUESE HIGHER EDUCATION STUDENTS

\begin{abstract}
In this article we investigate approaches to study, conceptions of learning, and teaching styles in Portuguese higher education students. It was our purpose to construct a Portuguese version of the Approaches and Study Skills Inventory for Students - short version (Tait, Entwistle \& McCune, 1998). Validity and reliability were considered and tested in a sample of Portuguese college students. The results from construct validity and internal consistency indicate that in general, the instrument measures the theoretical constructs. College students that integrated the studied sample did reveal certain ways of approaching the study and learning tasks. Nevertheless, we highlight some limitations, not only typical of instruments of this nature (auto-response), but also related to some procedures. In particular, we emphasize some less satisfactory results in some of the subscales of the inventory.
\end{abstract}

Keywords

Study approaches; Conceptions of learning; College students; ASSIST 


\section{ÉTUDE DE TRADUCTION, ADAPTATION ET VALIDATION DE L'ASSIST DANS UN ÉCHANTILLON D'ÉTUDIANTS UNIVERSITAIRES PORTUGAIS}

\section{Résumé}

Dans cette investigation, nous avons examiné comment les étudiants universitaires apprennent, étudient et quelles sont leur préférences concernant les styles d'enseignement. Notre référence a été le travail qu'Entwistle et ses collègues développent depuis plusieurs années. Notre objectif était de construire une version Portugaise du questionnaire Approaches and Study Skills Inventory for Students - short version (Tait, Entwistle \& McCune, 1998), supporté par plusieurs analyses pour évaluer la scientificité de l'inventaire. Les résultats nous permettent d'affirmer que, globalement, l'instrument peut être utilisé pour évaluer les conceptions théoriques postulées par l'auteur du questionnaire. Les étudiants universitaires qui ont fait partie de notre étude parait démontrer plusieurs conceptions et styles d'apprendre et d'étudier. Cependant les résultats obtenus, nous avons aussi observé quelques limitations concernant l'utilisation d'instruments d'auto-réponse comme l'ASSIST, et aussi résultants de quelques procédures utilisées. En particulier, nous pensons que certains résultats moins satisfacteurs relatives à certaines scalas du questionnaire, doivent être analysés avec prudence.

Mots-clé

Conceptions d'apprentissage, Étude, Étudiants universitaires, ASSIST

Recebido em Setembro/2008

Aceite para publicação em Abril/2009

Toda a correspondência relativa a este artigo deve ser enviada para: Sandra Cristina Andrade Teodósio dos Santos Valadas, Universidade do Algarve, Faculdade de Ciências Humanas e Sociais, Departamento de Ciências da Educação e Sociologia, Campus de Gambelas, 8005-139 Faro, Portugal. Telef.: +351289800914 (ext. 7628); Fax: +351289 800067; e-mail: svaladas@ualg.pt 